\title{
Morpho-Physiological Response and Production Potential of Promising Mungbean Cultivars under Varying Planting Dates
}

\author{
Abdul Mannan", Muhammad Bilal Chattha and Mubeen Sarwar \\ Institute of Agricultural Sciences, University of the Punjab, Lahore, Pakistan \\ *For correspondence: manich65@gmail.com \\ Received 10 September 2020; Accepted 31 December 2020; Published 25 January 2021
}

\begin{abstract}
Optimization of planting dates under any climatic conditions is pre-requisite to improve the yield and quality of the major and minor crops. This study was conducted to evaluate the potential of various mungbean cultivars for morpho-physiological and yield traits under different planting dates. In this pot study twenty mungbean cultivars (MGP-17, DM-D4, C5/95-3-31, C6/953-8, 5-63-94, TM-1407, MGP-01, NM-11, MGP-41, 5-63-1, MGP-16, NM20-21, MUNG-88, NM-121-25, RAMZAN, NM2016, NM-19-19, 1099, NM-51 and NM13-1) were planted on July 01 and August 01. Results revealed that various planting dates significantly affected all the attributes, however, genotypic variation was observed among the cultivars. Delayed planting reduced the stand establishment attributes as mean germination time (1.90\%), germination index $(3.10 \%)$, final germination percentage (7.34\%), seedling growth including shoot length $(14.88 \%)$, root length $(23.31 \%)$, number of leaves $(23.04 \%)$, leaf area $(5.74 \%)$ and number of nodules (13.02\%). Likely, gas exchange traits including photosynthetic rate (15.71\%), transpiration rate $(17.09 \%)$, sub-stomatal $\mathrm{CO}_{2}$ concentration $(2.39 \%)$, stomatal conductance $(30.56 \%)$, SPAD chlorophyll contents $(7.42 \%)$ and water use efficiency (3.28\%) were also reduced. Among morphological traits, various planting dates also reduced the number of pods per plant (5.04\%), length of the pod (5.69\%), number of grains per pod (28.68\%) and 1000-grains weight $(7.05 \%)$. Differential responses of all the mungbean cultivars were observed for all the pragmatic traits. Delayed planting significantly reduced the morpho-physiological and yield attributes of all mungbean cultivars. However, two mungbean cultivars (NM-121-25 and NM-2016) relatively performed better with minimum reductions in growth, yield and physiological attributes even in delayed planting while the DM-D4 and TM-1407 were found to be the most sensitive in delayed planting than other tested cultivars. Therefore, mungbean cultivars NM-121-25 and NM-2016 can be sown in late sown conditions to get higher yield. (C) 2021 Friends Science Publishers
\end{abstract}

Keywords: Planting dates; Yield; Leaf area; Delayed planting; Physiological attributes

\section{Introduction}

Mungbean (Vigna radiata L.) is an important annual, herbaceous and leguminous primordial plant belongs to family Fabaceae grown as a spring and summer crop in tropical and subtropical areas of the world (Miklas and Singh 2007; Chauhan et al. 2010). The genus Vigna has been extended to include about 150 species; among which 22 are native to India and 16 to Southeast Asia and remaining species are originated in Africa (Aditya and Jitendra 2011). India is the primary gene center of diversity and probable center of domestication of mungbean (Nassar 2003).

Potential yield of mungbean can be achieved through optimum use of inputs and agronomic practices. Besides other inputs, planting dates and improved cultivars are of primary importance (Ali and Gupta 2012). Singh et al. (2010) suggested that planting date is the most important non-monetary input to obtain optimum yield from mungbean. Similarly, Sadeghipour (2008) and Miah et al. (2009) stated that too early sowing may result in poor germination and poor plant stands, while yield from very late sown crop may be low due to unfavorable agro-climatic conditions for the growth and development of mungbean.

Selection of superior genotypes possessing better heritability and genetic advance for various traits is the prerequisite for achieving the maximum mungbean productivity. The yield can be increased to a greater extent by identifying high yielding cultivars and suitable planting date (Singh et al. 2010; Ali and Gupta 2012; (Hussain et al. 2012a, b).). Similarly, Naveed et al. (2015) indicated that optimum planting date is an important factor for achieving improved mungbean production in different agro-ecological zones of the world. In Pakistan the mostly the farmers of rainfed areas cultivate the mungbean for achieving the maximum yield. A good number of high yielding mungbean cultivars are available now in Pakistan but, farmers generally grow the local cultivars using minimum nutrients 
application and they rarely maintain the optimum planting time. Moreover, due to low income per unit of resources invested farmers are losing interest in producing mungbean. Therefore, attention should be given to increasing yield through selection of suitable cultivars and adoption of improved cultural practices for establishing mungbean as a profitable crop.

Delayed planting reduced the number of pods per plant and test weight of mungbean. The denaturation and/or aggregation of proteins with concomitant increase in fluidity of cell membrane lipids are the direct harms caused by temperature due to delayed planting (Howarth 2005). Although, interaction of planting time and mungbean cultivars has already been documented, however information regarding germination, seedling growth including physiological and yield attributes of available mungbean cultivar(s) at various planting time need to be explored. Therefore, this study was conducted with the hypothesis that delayed planting had negative effect on germination, growth, gas exchange traits and yield related traits of mungbean; however, the different cultivars might behave differently due to their divergent genetic makeup.

\section{Materials and Methods}

\section{Experimental site and climate}

This wire house study was executed at experimental area of Institute of Agricultural Sciences, University of the Punjab, Lahore, Pakistan. The experimental site is in subtropical climate region, with mean temperatures ranging from $6^{\circ} \mathrm{C}$ to $30^{\circ} \mathrm{C}$ in winter and from $27^{\circ} \mathrm{C}$ to $45^{\circ} \mathrm{C}$ in summer. The average annual rainfall is around $300 \mathrm{~mm}$, half of which is recorded between July and August as monsoons; and the weather data during the growth period is given in Fig. 1.

\section{Experimental Details}

Seeds of 20 mungbean cultivars (MGP-17, DM-D4, C5/953-31, C6/95-3-8, 5-63-94, TM-1407, MGP-01, NM-11, MGP-41, 5-63-1, MGP-16, NM20-21, MUNG-88, NM121-25, RAMZAN, NM-2016, NM-19-19, 1099, NM-51 and NM13-1) were collected from Plant Genetic Resource Institute (PGRI), National Agricultural Research Centre (NARC), Islamabad, Pakistan. Twenty above mentioned mungbean cultivars were sown on July 01 and on August 01, 2017. This experiment was planned in completely randomized design (CRD) with factorial arrangement with three replications.

\section{Crop management}

The planned trial was conducted in $9 \mathrm{~L}$ pots having dimensions $(45 \mathrm{~cm} \times 30 \mathrm{~cm}$ diameter $)$ allocating three pots for each treatment. Each pot was filled with $7 \mathrm{~kg}$ sand as growth media. The crop was sown on $1^{\text {st }}$ July and $1^{\text {st }}$ August, 2017 using ten seeds per pot. After seed germination, five seedlings of equal size were maintained per pot. Plants were fed with essential nutrients by supplying Hoagland's nutrient solution (full strength) after germination. After 10 days nutrient solution was changed and was continued until maturity. Irrigation was applied as per requirement of crop and hand weeding was done to avoid weed crop competation. Mature crop was harvested on Oct 12 and Nov 15, 2017.

\section{Observations, Measurements and Data Analysis}

Stand Establishement: Data for the stand establishment was calculated and the seedlings were counted daily after emergence to determine stand establishment traits by using Handbook AOSA (1990).

The mean emergence time was computed by using the formula given by Ellis and Robert (1981);

$$
\text { Mean emergence time }=\frac{\Sigma D n}{\Sigma n}
$$

Where, $\mathrm{n}=$ Seedlings emerged on day $\mathrm{D} ; \mathrm{D}=$ Days from initiation of the emergence.

Germination index was determined using the formula of Association of Official Seed Analyst (1990).

Emergence index $=\frac{\text { No. of emerged seedlings }}{\text { day of first count }}+\cdots+\frac{\text { No. of emerged seedlings }}{\text { day of final count }}$

While the emergence percentage of final count was computed as a ratio of the seedlings emerged to the total seeds sown and expressed in percentage.

\section{Growth attributes}

At the end of experiment, 90 days after sowing (DAS) the shoot length and root length of selected plants was measured using measuring scale and expressed in $\mathrm{cm}$. Shoot fresh weight and root fresh weight of selected plants was weighed with electric weighing balance after separating roots and shoots then expressed in gram (g). While for the dry weights shoots and roots of all selected plants were dried in oven at $70^{\circ} \mathrm{C}$, expressed in grams $(\mathrm{g})$. Leaves of the selected mungbean plants were counted from each pot and the average was taken. Leaves of three selected plant from all replications were detached and the leaf area was determined with digital leaf area meter. After pulling out the selected plants from the sand, number of nodules were counted and then averaged.

\section{Physiological attributes}

Stomatal conductance $\left(\mathrm{g}_{\mathrm{s}}\right)$, sub-stomatal $\mathrm{CO}_{2}$ concentration (Ci), photosynthetic rate $(\mathrm{A})$, transpiration rate $(\mathrm{E})$ and leaf temperature was measured on the 45 DAS on fully expanded upper most leaves with portable photosynthesis system (Infra-Red Gas Analyzer) at light saturating intensity between 9:00am to 12:00 noon while water use efficiency was calculated by applying the formula $(\mathrm{A} / \mathrm{E})$, while the 


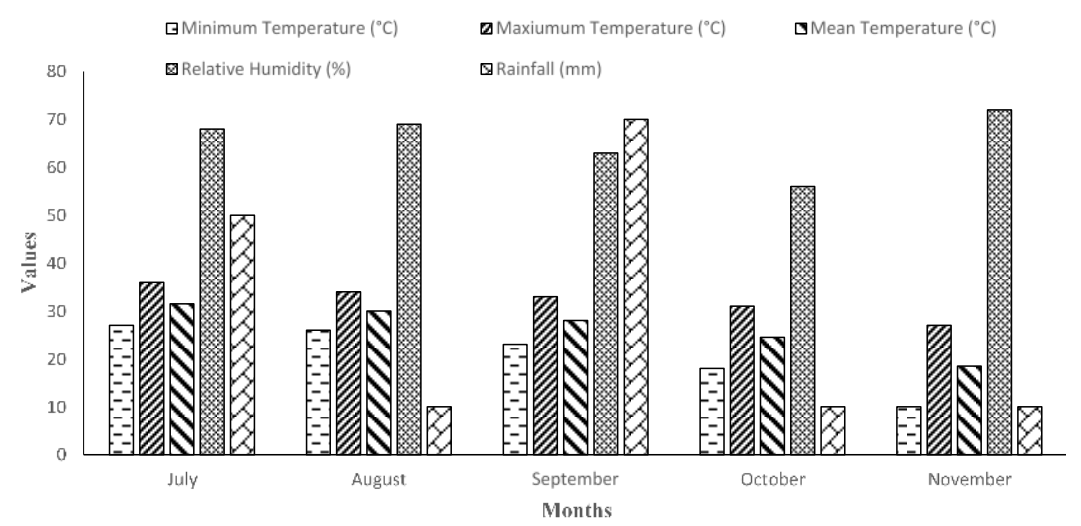

Fig. 1: Weather data during the crop growth cycle

Table 1: Effect of planting dates on germination attributes of mungbean cultivars

\begin{tabular}{|c|c|c|c|}
\hline Treatments & MGT (Days) & GI & FGP $(\%)$ \\
\hline \multicolumn{4}{|l|}{ Planting dates (PD) } \\
\hline July 01 & $5.25 \mathrm{~A}$ & $4.20 \mathrm{~A}$ & $81.78 \mathrm{~A}$ \\
\hline August 01 & $5.15 \mathrm{~B}$ & $4.07 \mathrm{~B}$ & $75.78 \mathrm{~B}$ \\
\hline HSD value at $\mathrm{p} \leq 0.01$ & 0.02 & 0.10 & 0.03 \\
\hline \multicolumn{4}{|l|}{ Mungbean cultivars (C) } \\
\hline MGP-17 & $5.37 \mathrm{GH}$ & 4.01 & $79.98 \mathrm{E}$ \\
\hline DM-D4 & $4.56 \mathrm{Q}$ & 2.48 & $71.99 \mathrm{~L}$ \\
\hline C5/95-3-31 & $4.78 \mathrm{MN}$ & 2.82 & $73.99 \mathrm{~J}$ \\
\hline C6/95-3-8 & 4.71 NO & 2.68 & $73.99 \mathrm{~J}$ \\
\hline $5-63-94$ & $4.66 \mathrm{OP}$ & 2.57 & $72.98 \mathrm{~K}$ \\
\hline TM-1407 & 4.61 PQ & 2.39 & $71.99 \mathrm{~L}$ \\
\hline MGP-01 & $5.30 \mathrm{HI}$ & 3.88 & $78.98 \mathrm{~F}$ \\
\hline NM-11 & $5.45 \mathrm{FG}$ & 4.16 & $79.99 \mathrm{E}$ \\
\hline MGP-41 & $5.26 \mathrm{IJ}$ & 3.74 & $78.98 \mathrm{~F}$ \\
\hline $5-63-1$ & 4.85 LM & 2.93 & $75.92 \mathrm{I}$ \\
\hline MGP-16 & $5.22 \mathrm{IJ}$ & 3.63 & $77.96 \mathrm{G}$ \\
\hline NM20-21 & $5.52 \mathrm{EF}$ & 4.30 & 81.97 D \\
\hline MUNG-88 & $4.92 \mathrm{~L}$ & 3.05 & $75.99 \mathrm{I}$ \\
\hline NM-121-25 & $5.83 \mathrm{~A}$ & 4.89 & $87.00 \mathrm{~A}$ \\
\hline RAMZAN & $5.17 \mathrm{JK}$ & 3.48 & $76.99 \mathrm{H}$ \\
\hline NM-2016 & $5.78 \mathrm{AB}$ & 4.73 & $86.99 \mathrm{~A}$ \\
\hline NM-19-19 & $5.70 \mathrm{BC}$ & 4.69 & $84.98 \mathrm{~B}$ \\
\hline 1099 & $5.10 \mathrm{~K}$ & 3.23 & $76.98 \mathrm{H}$ \\
\hline NM-51 & $5.64 \mathrm{CD}$ & 4.55 & $84.02 \mathrm{C}$ \\
\hline NM13-1 & $5.57 \mathrm{DE}$ & 4.43 & $84.01 \mathrm{C}$ \\
\hline HSD value at $\mathrm{p} \leq 0.01$ & 0.09 & 0.09 & 0.20 \\
\hline Significance Level (PD) & $* *$ & $* *$ & $* *$ \\
\hline Significance Level (C) & $* *$ & NS & $* *$ \\
\hline Significance Level $(\mathrm{PD} \times \mathrm{C})$ & NS & NS & NS \\
\hline
\end{tabular}

SPAD chlorophyll values were measured by SPAD 502 Plus Chlorophyll Meter.

\section{Yield attributes}

All the yield attributes such as pods per plant, length of pod, grains per pod and 1000 grains weight were measured by taking three randomly selected plants of each pot following the protocols of Haider et al. (2020).

\section{Statistical analysis}

Data were analysed using software Statistix 8.1 (Analytical Computer Software, Statistix 8.1; Tallahassee, F.L., U.S.A., 1985-2003) following two-way ANOVA under CRD with factorial arrangement. In case of significance, Highest Significance Difference (HSD) test at 1\% probability level was used to seprate treatments means (Steel et al. 1997).

\section{Results}

\section{Germination and seedling growth attributes}

Various planting dates and mungbean cultivars showed significant variation $(p \leq 0.01)$ for mean germination time, germination index and final germination percentage (Table 1). Both the factors exhibited significant results except for cultivars in germination index, while the interactive effect of $\mathrm{PD} \times \mathrm{C}$ was non-significant for germination attributes. For planting dates, reduction in mean germination time $(1.94 \%)$, germination index $(3.09 \%)$ and final germination percentage (7.33\%) was observed when mungbean cultivars were sown on $1^{\text {st }}$ July as compared to August sowing. Among mungbean cultivars, maximum mean germination time (5.83 days) and final germination percentage $(87.00 \%)$ was observed in NM-121-25 followed by in NM-2016 and NM19-19 which are statistically similar with each other. While minimum mean germination time (4.61 days) and final germination percentage (71.99) was observed in TM-1407.

Planting dates and mungbean cultivars had significant variation $(p \leq 0.01)$ for seedling growth attributes (Table 2 ) except for number of leaves for planting dates effects and number of nodules for mungbean cultivars had nonsignificant effects. However, the interactions for all the growth attributes were non-significant. Delayed planting $\left(1^{\text {st }}\right.$ August) significantly impaired the growth of entire mung bean cultivars. Reduction in shoot length $(14.87 \%)$, root length $(23.31 \%)$, number of leaves $(23.04 \%)$, shoot dry weight $(8.58 \%)$, root dry weight $(13.22 \%)$ and leaf area $(5.74 \%)$ was observed when mungbean cultivars were sown 
Table 2: Effect of planting dates on growth attributes of mungbean cultivars

\begin{tabular}{|c|c|c|c|c|c|c|c|}
\hline Treatments & $\begin{array}{l}\text { Shoot length } \\
(\mathrm{cm})\end{array}$ & $\begin{array}{l}\text { Root length } \\
(\mathrm{cm})\end{array}$ & $\begin{array}{l}\text { Number of leaves } \\
\text { plant }^{-1}\end{array}$ & $\begin{array}{l}\text { Shoot DW (g } \\
\left.\text { plant }^{-1}\right)\end{array}$ & $\begin{array}{l}\text { Root DW (g } \\
\left.\text { plant }^{-1}\right)\end{array}$ & $\begin{array}{l}\text { Number of nodules } \\
\text { plant }^{-1}\end{array}$ & $\begin{array}{l}\text { LA }\left(\mathrm{cm}^{2}\right. \\
\left.\text { plant }^{-1}\right)\end{array}$ \\
\hline \multicolumn{8}{|l|}{ Planting dates (PD) } \\
\hline July 01 & $51.76 \mathrm{~A}$ & $31.74 \mathrm{~A}$ & $8.68 \mathrm{~A}$ & $6.41 \mathrm{~A}$ & $2.42 \mathrm{~A}$ & 7.68 & $131.26 \mathrm{~A}$ \\
\hline August 01 & $44.06 \mathrm{~B}$ & $24.34 \mathrm{~B}$ & $6.68 \mathrm{~B}$ & $5.86 \mathrm{~B}$ & $2.10 \mathrm{~B}$ & 6.68 & $123.72 \mathrm{~B}$ \\
\hline HSD value at $p \leq 0.01$ & 0.17 & 0.16 & 1.30 & 0.01 & 0.01 & NS & 0.01 \\
\hline \multicolumn{8}{|l|}{ Mungbean cultivars (C) } \\
\hline MGP-17 & $49.71 \mathrm{GH}$ & $29.66 \mathrm{GH}$ & 8.66 & $6.47 \mathrm{H}$ & $2.50 \mathrm{E}$ & $6.83 \mathrm{AB}$ & $134.68 \mathrm{G}$ \\
\hline DM-D4 & $41.95 \mathrm{R}$ & $21.93 \mathrm{QR}$ & 4.66 & $4.81 \mathrm{R}$ & $1.64 \mathrm{~N}$ & $4.16 \mathrm{AB}$ & $106.05 \mathrm{R}$ \\
\hline C5/95-3-31 & $44.48 \mathrm{OP}$ & $24.56 \mathrm{O}$ & 6.33 & $5.34 \mathrm{O}$ & $1.80 \mathrm{KL}$ & $5.83 \mathrm{AB}$ & $112.47 \mathrm{O}$ \\
\hline C6/95-3-8 & 43.55 PQ & $22.66 \mathrm{OP}$ & 7.33 & $5.18 \mathrm{P}$ & $1.75 \mathrm{LM}$ & $5.16 \mathrm{AB}$ & $110.61 \mathrm{P}$ \\
\hline $5-63-94$ & $42.91 \mathrm{QR}$ & $22.73 \mathrm{PQ}$ & 7.00 & $4.93 \mathrm{Q}$ & $1.69 \mathrm{MN}$ & $3.50 \mathrm{AB}$ & 107.73 Q \\
\hline TM-1407 & $40.58 \mathrm{~S}$ & $21.03 \mathrm{R}$ & 5.33 & $4.61 \mathrm{~S}$ & $1.55 \mathrm{O}$ & $2.83 \mathrm{~B}$ & $102.90 \mathrm{~S}$ \\
\hline MGP-01 & $49.01 \mathrm{HI}$ & $30.96 \mathrm{HI}$ & 8.33 & $6.34 \mathrm{I}$ & $2.38 \mathrm{~F}$ & $8.16 \mathrm{AB}$ & $131.79 \mathrm{H}$ \\
\hline NM-11 & $50.28 \mathrm{FG}$ & $30.46 \mathrm{FG}$ & 9.00 & $6.57 \mathrm{G}$ & $2.63 \mathrm{D}$ & $7.83 \mathrm{AB}$ & $136.91 \mathrm{~F}$ \\
\hline MGP-41 & $48.35 \mathrm{IJ}$ & $28.33 \mathrm{IJ}$ & 6.33 & $6.23 \mathrm{~J}$ & $2.24 \mathrm{G}$ & $7.50 \mathrm{AB}$ & $129.79 \mathrm{I}$ \\
\hline $5-63-1$ & $45.11 \mathrm{KL}$ & $25.56 \mathrm{~N}$ & 6.66 & $5.57 \mathrm{~N}$ & $1.84 \mathrm{JK}$ & $7.83 \mathrm{AB}$ & $115.56 \mathrm{~N}$ \\
\hline MGP-16 & $47.58 \mathrm{JK}$ & $27.70 \mathrm{JK}$ & 6.33 & $6.15 \mathrm{~J}$ & $2.18 \mathrm{G}$ & $6.50 \mathrm{AB}$ & $126.95 \mathrm{~J}$ \\
\hline NM20-21 & $50.91 \mathrm{EF}$ & $31.13 \mathrm{EF}$ & 9.33 & $6.72 \mathrm{~F}$ & $2.68 \mathrm{D}$ & $8.83 \mathrm{AB}$ & $139.70 \mathrm{E}$ \\
\hline MUNG-88 & $45.78 \mathrm{MN}$ & $26.03 \mathrm{MN}$ & 6.66 & $5.71 \mathrm{M}$ & $1.90 \mathrm{IJ}$ & $5.83 \mathrm{AB}$ & $118.77 \mathrm{M}$ \\
\hline NM-121-25 & $54.28 \mathrm{~A}$ & $34.46 \mathrm{~A}$ & 11.00 & $7.57 \mathrm{~A}$ & $2.98 \mathrm{~A}$ & $11.16 \mathrm{~A}$ & $148.31 \mathrm{~A}$ \\
\hline RAMZAN & $47.11 \mathrm{KL}$ & $27.00 \mathrm{JK}$ & 6.66 & $6.02 \mathrm{~K}$ & $2.04 \mathrm{H}$ & $5.50 \mathrm{AB}$ & $124.11 \mathrm{~K}$ \\
\hline NM-2016 & $53.68 \mathrm{AB}$ & $33.70 \mathrm{AB}$ & 11.00 & $7.47 \mathrm{~B}$ & $2.94 \mathrm{AB}$ & $10.83 \mathrm{~A}$ & $148.24 \mathrm{~A}$ \\
\hline NM-19-19 & $52.98 \mathrm{BC}$ & $33.13 \mathrm{BC}$ & 10.33 & $7.24 \mathrm{C}$ & $2.89 \mathrm{AB}$ & $9.50 \mathrm{AB}$ & $146.99 \mathrm{~B}$ \\
\hline 1099 & $46.35 \mathrm{LM}$ & $26.60 \mathrm{LM}$ & 6.66 & $5.87 \mathrm{~L}$ & $1.95 \mathrm{HI}$ & $7.16 \mathrm{AB}$ & $120.49 \mathrm{~L}$ \\
\hline NM-51 & $52.25 \mathrm{CD}$ & $32.36 \mathrm{CD}$ & 8.33 & $7.09 \mathrm{D}$ & $2.85 \mathrm{BC}$ & $9.83 \mathrm{AB}$ & $144.58 \mathrm{C}$ \\
\hline NM13-1 & $51.38 \mathrm{DE}$ & $31.1373 \mathrm{DE}$ & 7.66 & $6.89 \mathrm{E}$ & $2.79 \mathrm{C}$ & $8.83 \mathrm{AB}$ & 143.20 D \\
\hline HSD value at $\mathrm{p} \leq 0.01$ & 1.01 & 0.94 & NS & 0.09 & 0.09 & 7.89 & 0.08 \\
\hline Significance Level (PD) & $* *$ & $* *$ & $* *$ & $* *$ & $* *$ & NS & ** \\
\hline Significance Level (C) & $* *$ & $* *$ & NS & $* *$ & $* *$ & $* *$ & $* *$ \\
\hline $\begin{array}{l}\text { Significance Level (PD } \\
\text { C) }\end{array}$ & NS & NS & NS & NS & NS & NS & NS \\
\hline
\end{tabular}

in the month of August. For mungbean cultivars, maximum shoot length $(54.28 \mathrm{~cm})$, root length $(34.46 \mathrm{~cm})$, shoot dry weight $(7.57 \mathrm{~g})$, root dry weight $(2.98 \mathrm{~g})$, leaf area (148.31 $\mathrm{cm}^{2}$ ) and number of nodules (11.16) was observed in NM121-25 followed by in NM-2016 and NM-19-19 respectively. While minimum shoot length $(40.58 \mathrm{~cm})$, root length $(21.03 \mathrm{~cm})$, shoot dry weight $(4.61 \mathrm{~g})$, root dry weight $(1.55 \mathrm{~g})$, leaf area $\left(102.90 \mathrm{~cm}^{2}\right)$ and number of nodules (2.83) was noticed in TM-1407 (40.58 cm) (Table 2).

\section{Gas exchange attributes and SPAD-chlorophyll values}

Different planting dates and mungbean cultivars showed significant variation $(p \leq 0.01)$ for gas exchange attributes, leaf temperature, water use efficiency and SPAD chlorophyll values (Table 3). Interestingly, the interactive effects were non-significant for all the attributes except the SPAD chlorophyll values. Reduction in photosynthetic rate $(15.71 \%)$, transpiration rate $(17.09 \%)$, sub-stomatal $\mathrm{CO}_{2}$ concentration $(2.38 \%)$, stomatal conductance to water $(30.55 \%)$, water use efficiency $(3.28 \%)$, SPAD chlorophyll contents $(7.42 \%)$ and increase in leaf temperature $(6.76 \%)$ was observed in when mungbean cultivars were sown in the month of August. For mungbean cultivars maximum photosynthetic rate $\left(37.65 \mu \mathrm{mol} \mathrm{mm} \mathrm{m}^{-2} \mathrm{~s}^{-1}\right)$, transpiration rate
(1.30 mmol m $\left.\mathrm{m}^{-2} \mathrm{~s}^{-1}\right)$, sub-stomatal $\mathrm{CO}_{2}$ concentration (312.21 $\mathrm{vpm})$, stomatal conductance to water $\left(0.41 \mathrm{mmol} \mathrm{m}^{-2} \mathrm{~s}^{-1}\right)$, leaf temperature $\left(38.45^{\circ} \mathrm{C}\right)$ in DM-D4, water use efficiency $\left(3.91 \mathrm{~kg} / \mathrm{ha} \mathrm{mm}^{-1}\right)$ and SPAD chlorophyll contents (43.94) was observed in NM-121-25 followed by in NM-2016 and NM-19-19 respectively. While minimum photosynthetic rate $\left(30.45 \mu \mathrm{mol} \mathrm{mm}^{-2} \mathrm{~s}^{-1}\right)$, transpiration rate $\left(0.84 \mathrm{mmol} \mathrm{m}^{-2}\right.$ $\left.\mathrm{s}^{-1}\right)$, sub-stomatal $\mathrm{CO}_{2}$ concentration (237.12 vpm), stomatal conductance to water $\left(0.21 \mathrm{mmol} \mathrm{m}^{-2} \mathrm{~s}^{-1}\right)$, leaf temperature $\left(35.00^{\circ} \mathrm{C}\right)$ in NM-121-25, water use efficiency $(1.90 \mathrm{~kg} / \mathrm{ha}$ $\mathrm{mm}^{-1}$ ) and SPAD chlorophyll contents (29.96) was noticed

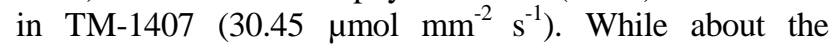
interactive effect of $\mathrm{PD} \times \mathrm{C}$ maximum SPAD chlorophyll contents (58.35) were observed in NM-121-25 followed by in NM-2016 (58.00) was observed in $\mathrm{PD}_{1}$ when mungbean cultivars were sown on $1^{\text {st }}$ July, while minimum SPAD chlorophyll contents were noticed in $\mathrm{PD}_{2}$ (49.18) where mungbean cultivars (TM-1407) were sown on $1^{\text {st }}$ August (Fig. 2).

\section{Yield attributes}

Both the factors planting dates and mungbean cultivars exhibited statistically significant effects for morphological attributes except for mungbean cultivars in grains per pod and pods per plant for sowing dates while the interactive 
Mannan et al. / Intl J Agric Biol, Vol 25, No 3, 2021

Table 3: Effect of planting dates on physiological attributes of mungbean cultivars

\begin{tabular}{|c|c|c|c|c|c|c|c|}
\hline Treatments & $\begin{array}{l}\text { PR } \\
\left(\mu \mathrm{mol} \mathrm{mm} \mathrm{m}^{-2} \mathrm{~s}^{-1}\right)\end{array}$ & $\begin{array}{l}\text { TR } \\
\left(\mathrm{mmol} \mathrm{m}{ }^{-2} \mathrm{~s}^{-1}\right)\end{array}$ & $\begin{array}{l}C i \\
(\mathrm{vpm})\end{array}$ & $\begin{array}{l}\text { SCW } \\
\left(\mathrm{mmol} \mathrm{mm}^{-2} \mathrm{~s}^{-1}\right)\end{array}$ & $\begin{array}{l}\text { LT } \\
\left({ }^{\circ} \mathrm{C}\right) \\
\end{array}$ & $\begin{array}{l}\text { WUE } \\
\left(\mathrm{kg} \mathrm{ha}^{-1} \mathrm{~mm}^{-1}\right)\end{array}$ & $\begin{array}{l}\text { SPAD } \\
\text { CC }\end{array}$ \\
\hline \multicolumn{8}{|l|}{ Planting dates (PD) } \\
\hline July 01 & $35.77 \mathrm{~A}$ & $1.17 \mathrm{~A}$ & $271.67 \mathrm{~A}$ & $0.36 \mathrm{~A}$ & $38.45 \mathrm{~B}$ & $2.74 \mathrm{~A}$ & $37.73 \mathrm{~A}$ \\
\hline August 01 & $30.15 \mathrm{~B}$ & $0.97 \mathrm{~B}$ & $265.18 \mathrm{~B}$ & $0.25 \mathrm{~B}$ & $41.05 \mathrm{~A}$ & $2.65 \mathrm{~B}$ & $34.93 \mathrm{~B}$ \\
\hline HSD value at $p \leq 0.01$ & 0.01 & 0.01 & 0.01 & 0.01 & 1.29 & 0.01 & 0.01 \\
\hline \multicolumn{8}{|l|}{ Mungbean cultivars (C) } \\
\hline MGP-17 & $33.52 \mathrm{H}$ & $1.10 \mathrm{D}-\mathrm{F}$ & $280.30 \mathrm{H}$ & $0.32 \mathrm{~B}-\mathrm{E}$ & $40.83 \mathrm{~A}-\mathrm{C}$ & $2.83 \mathrm{H}$ & $36.94 \mathrm{H}$ \\
\hline DM-D4 & $30.63 \mathrm{~S}$ & $0.88 \mathrm{KL}$ & $239.64 \mathrm{~S}$ & $0.22 \mathrm{G}$ & $43.83 \mathrm{~A}$ & $1.95 \mathrm{PQ}$ & $30.12 \mathrm{R}$ \\
\hline C5/95-3-31 & $31.23 \mathrm{P}$ & $0.97 \mathrm{H}-\mathrm{J}$ & $244.12 \mathrm{P}$ & $0.23 \mathrm{G}$ & $40.16 \mathrm{AB}$ & $2.13 \mathrm{MN}$ & $32.74 \mathrm{O}$ \\
\hline C6/95-3-8 & $31.00 \mathrm{Q}$ & $0.94 \mathrm{I}-\mathrm{K}$ & $242.25 \mathrm{Q}$ & $0.22 \mathrm{G}$ & $39.83 \mathrm{~A}-\mathrm{C}$ & $2.06 \mathrm{NO}$ & $32.60 \mathrm{P}$ \\
\hline $5-63-94$ & $30.84 \mathrm{R}$ & $0.91 \mathrm{~J}-\mathrm{L}$ & $240.36 \mathrm{R}$ & $0.21 \mathrm{G}$ & $43.16 \mathrm{AB}$ & $2.00 \mathrm{OP}$ & $30.38 \mathrm{Q}$ \\
\hline TM-1407 & $30.45 \mathrm{~T}$ & $0.84 \mathrm{~L}$ & $237.12 \mathrm{~T}$ & $0.21 \mathrm{G}$ & $43.16 \mathrm{AB}$ & $1.90 \mathrm{Q}$ & $29.96 \mathrm{~S}$ \\
\hline MGP-01 & $32.96 \mathrm{I}$ & $1.07 \mathrm{E}-\mathrm{G}$ & $271.38 \mathrm{I}$ & 0.34 A-D & $40.50 \mathrm{~A}-\mathrm{C}$ & $2.70 \mathrm{I}$ & $36.60 \mathrm{I}$ \\
\hline NM-11 & $33.85 \mathrm{G}$ & 1.14 C-E & $282.03 \mathrm{G}$ & 0.34 A-D & $40.83 \mathrm{~A}-\mathrm{C}$ & $2.95 \mathrm{G}$ & $37.37 \mathrm{G}$ \\
\hline MGP-41 & $32.63 \mathrm{~J}$ & $1.06 \mathrm{E}-\mathrm{G}$ & $266.34 \mathrm{~J}$ & $0.32 \mathrm{~B}-\mathrm{E}$ & $40.83 \mathrm{~A}-\mathrm{C}$ & $2.57 \mathrm{~J}$ & $35.41 \mathrm{~J}$ \\
\hline $5-63-1$ & $31.40 \mathrm{O}$ & $1.00 \mathrm{G}-\mathrm{J}$ & $245.50 \mathrm{O}$ & $0.23 \mathrm{FG}$ & $42.83 \mathrm{AB}$ & $2.21 \mathrm{M}$ & $32.80 \mathrm{O}$ \\
\hline MGP-16 & $32.19 \mathrm{~K}$ & $1.04 \mathrm{~F}-\mathrm{H}$ & $261.56 \mathrm{~K}$ & $0.31 \mathrm{~B}-\mathrm{E}$ & $41.50 \mathrm{~A}-\mathrm{C}$ & $2.50 \mathrm{JK}$ & $35.10 \mathrm{~K}$ \\
\hline NM20-21 & $34.00 \mathrm{~F}$ & $1.16 \mathrm{CD}$ & $284.51 \mathrm{~F}$ & $0.35 \mathrm{~A}-\mathrm{C}$ & $39.50 \mathrm{~A}-\mathrm{C}$ & $3.11 \mathrm{~F}$ & $40.23 \mathrm{~F}$ \\
\hline MUNG-88 & $31.58 \mathrm{~N}$ & $1.02 \mathrm{~F}-\mathrm{I}$ & $248.95 \mathrm{~N}$ & $0.25 \mathrm{E}-\mathrm{G}$ & $36.16 \mathrm{BC}$ & $2.21 \mathrm{M}$ & $32.99 \mathrm{~N}$ \\
\hline NM-121-25 & $37.65 \mathrm{~A}$ & $1.30 \mathrm{~A}$ & $312.21 \mathrm{~A}$ & $0.41 \mathrm{~A}$ & $35.00 \mathrm{C}$ & $3.91 \mathrm{~A}$ & $43.94 \mathrm{~A}$ \\
\hline RAMZAN & $31.91 \mathrm{~L}$ & $1.01 \mathrm{G}-\mathrm{I}$ & $257.28 \mathrm{~L}$ & $0.30 \mathrm{C}-\mathrm{F}$ & $36.66 \mathrm{~A}-\mathrm{C}$ & $2.42 \mathrm{~K}$ & $34.60 \mathrm{~L}$ \\
\hline NM-2016 & $36.86 \mathrm{~B}$ & $1.27 \mathrm{AB}$ & $309.53 \mathrm{~B}$ & $0.39 \mathrm{AB}$ & $36.50 \mathrm{~A}-\mathrm{C}$ & $3.77 \mathrm{~B}$ & $43.79 \mathrm{~B}$ \\
\hline NM-19-19 & $35.91 \mathrm{C}$ & $1.25 \mathrm{AB}$ & $304.42 \mathrm{C}$ & $0.36 \mathrm{~A}-\mathrm{C}$ & $36.83 \mathrm{~A}-\mathrm{C}$ & $3.66 \mathrm{C}$ & $43.60 \mathrm{C}$ \\
\hline 1099 & $31.78 \mathrm{M}$ & 0.99 G-J & $253.26 \mathrm{M}$ & $0.27 \mathrm{D}-\mathrm{G}$ & $39.83 \mathrm{~A}-\mathrm{C}$ & $2.32 \mathrm{~L}$ & $33.21 \mathrm{M}$ \\
\hline NM-51 & $34.57 \mathrm{D}$ & $1.21 \mathrm{BC}$ & $296.58 \mathrm{D}$ & $0.36 \mathrm{~A}-\mathrm{C}$ & $38.83 \mathrm{~A}-\mathrm{C}$ & $3.45 \mathrm{D}$ & $42.34 \mathrm{D}$ \\
\hline NM13-1 & $34.57 \mathrm{E}$ & $1.19 \mathrm{BC}$ & $291.15 \mathrm{E}$ & $0.35 \mathrm{~A}-\mathrm{C}$ & $38.16 \mathrm{~A}-\mathrm{C}$ & $3.30 \mathrm{E}$ & $41.97 \mathrm{E}$ \\
\hline HSD value at $p \leq 0.01$ & 0.07 & 0.08 & 0.08 & 0.07 & 7.52 & 0.08 & 0.08 \\
\hline Significance Level (PD) & $* *$ & $* *$ & $* *$ & $* *$ & $* *$ & $* *$ & $* *$ \\
\hline Significance Level (C) & ** & ** & $* *$ & $* *$ & $* *$ & $* *$ & $* *$ \\
\hline Significance Level $(\mathrm{PD} \times \mathrm{C})$ & NS & NS & NS & NS & NS & NS & ** \\
\hline
\end{tabular}

Means following same letters, within a column, are not statistically different from each other at $\mathrm{p} \leq 0.01$ according to HSD test

$\mathrm{PR}=$ Photosynthetic rate; TR $=$ Transpiration rate; $\mathrm{Ci}=$ Sub-stomatal $\mathrm{CO}_{2}$ concentration; $\mathrm{SCW}=\mathrm{Stomatal}$ conductance to water; $\mathrm{LT}=\mathrm{Leaf}$ temperature; WUE $=\mathrm{Water}$ use efficiency; SPAD CC $=$ SPAD chlorophyll contents

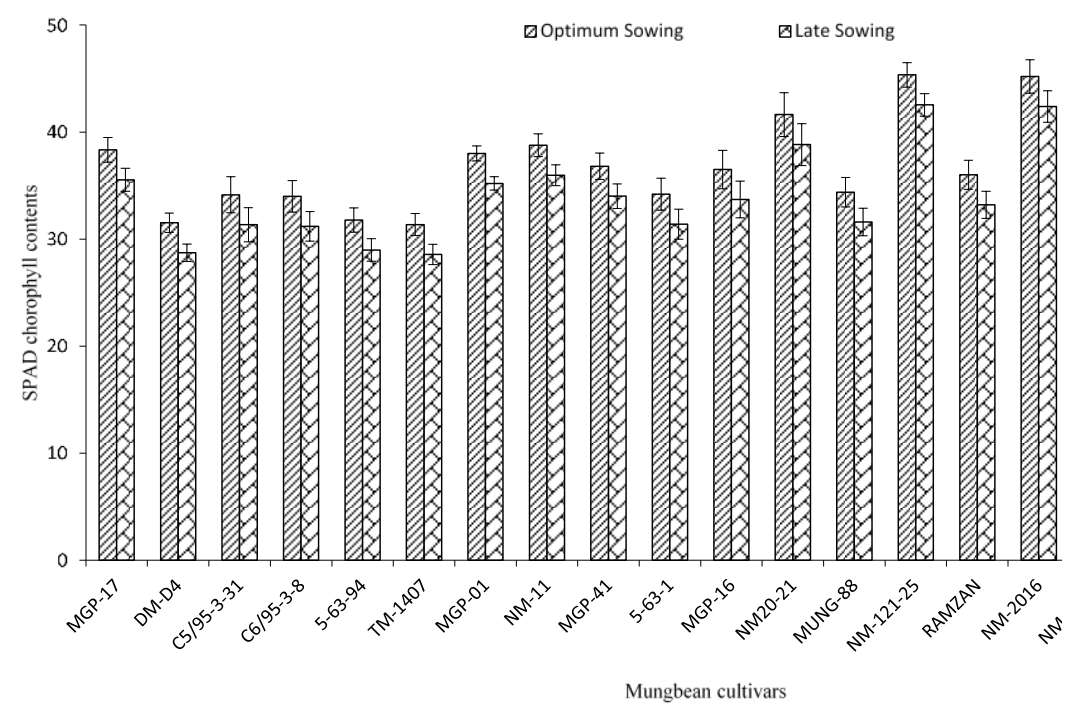

Fig. 2: Interactive effect of planting time and mungbean cultivars on SPAD chlorophyll contents of mungbean Optimum sowing $=$ July 01 ; Late sowing $=$ August 01

effect of $\mathrm{PD} \times \mathrm{C}$ also had non-significant response for all yield attributes except for the 1000-grains weight. Reduction in pod length $(6.03 \%)$, grains per pod $(28.80 \%)$ and 1000 grains weight $(7.56 \%)$ was observed in when mungbean cultivars were sown in the month of August. For mungbean cultivars maximum number of pods per plant (25.83), pod length $(11.58 \mathrm{~cm})$ and 1000 grains weight (56.47 g) were observed in NM-121-25 followed by in NM2016 and NM-19-19. While minimum number of pods per plant (10.16), pod length $(5.81 \mathrm{~cm})$ and 1000 grains weight 


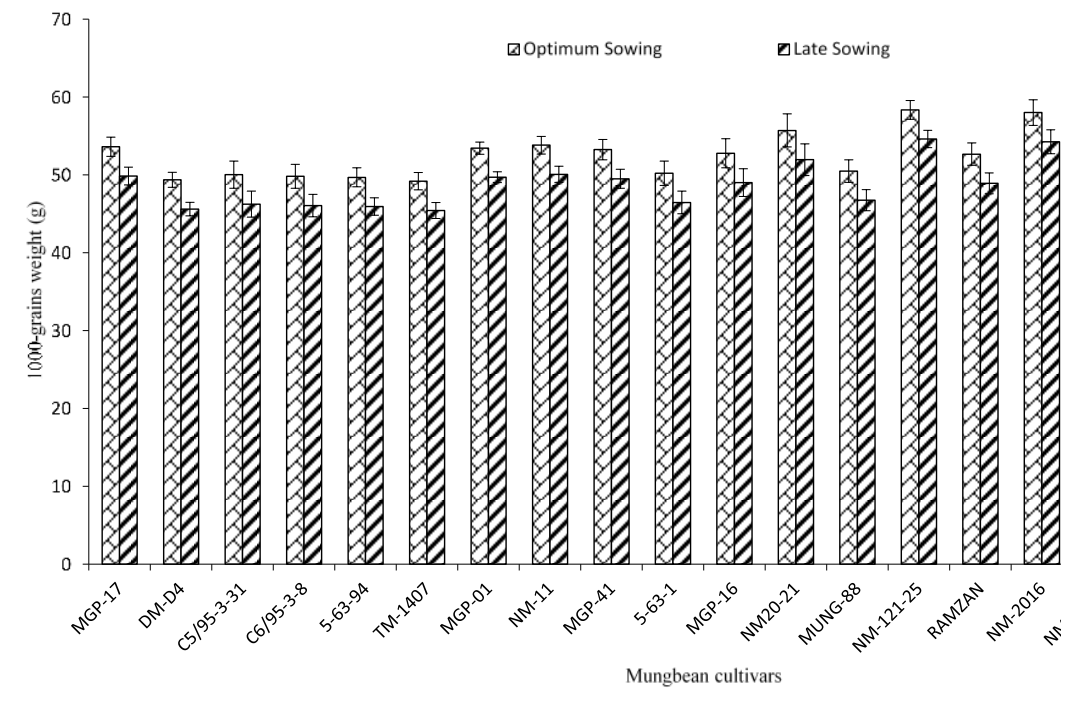

Fig. 3: Interactive effect of planting dates and mungbean cultivars on 1000-grains weight ( $\mathrm{g}$ ) of mungbean Optimum sowing= July 01 ; Late sowing $=$ August 01

$(47.30 \mathrm{~g})$ were noticed in TM-1407. Among the interactions, maximum 1000 grains weight was observed in NM-121-25 followed by in NM-2016 was observed in $\mathrm{PD}_{1}$ when mungbean cultivars were sown on $1^{\text {st }}$ July, while minimum 1000 grains weight was noticed in $\mathrm{PD}_{2}$ where mungbean cultivars (TM-1407) were sown on $1^{\text {st }}$ August (Fig. 3).

\section{Discussion}

Selection of superior parents is a prerequisite for any yield improvement program (Ahmad et al. 2008). Planting time, a non-monetary input, is the single most important factor to obtain optimum yield from mungbean (Sadeghipour 2008; Sarwar et al. 2019). So, determination of optimum planting time for mungbean is inevitable. Best time of planting of mungbean may vary from variety to variety and season to season due to variation in agroecological conditions (Ramakrishna et al. 2000; Reddy 2009). Delayed sowing reduces yield of summer mungbean (Palsaniya et al. 2016; Khanum et al. 2019). It was also described earlier that different genotypes may revealed significant variation under various environmental conditions and results of the current research are parallel with previous findings (Abdelmageed and Gruda 2009).

In this study, result showed that late planting had an adverse effect on the germination and growth attributes of mungbean including mean germination time, germination index, final germination percentage (Table 1), root and shoot length, shoot and root dry weight, leaf area per plant, number of leaves per plant as compared to optimum planting time (Table 2). Similar confirmation of findings had been reported in field crops and vegetables under control environmental conditions (Ashraf and Harris 2013).
At environmental conditions, number of genotypes did not show positive growth response compared to others (Ali et al. 2014; Campbell et al. 2019). With vigorous growth under variable environmental conditions, tolerant genotypes showed their ability to withstand under variation in environmental conditions due to delayed planting compared to sensitive ones with significantly less growth reduction (Vorasoot et al. 2003; Thakur et al. 2010). Observations of this study, illustrated that some of the genotypes studied did not gave satisfactory growth comparable to the rest.

It has been examined in present screening experiment that delayed planting of mungbean genotypes showed reasonable growth index, while some of them gave poor performance as demonstrated by other researchers (Naika $e t$ al. 2005). There were significant differences among growth variables. Those genotypes which reveal vigorous growth than others signified their capacity to tolerate the adverse environmental conditions. Similar findings were also noticed in a study where genetic characterization of mungbean genotypes against variation in the atmospheric conditions was done (Uddin et al. 2014). In present research, leaf number was considered as positive variable which specifies that the genotypes possessed a greater number of leaves under delayed planting revealed higher photosynthetic rate and hence increased growth rate with variation in the ambient temperature (Hussain et al. 2007; Asseng et al. 2011; Gezer 2018). Phurailatpam et al. (2007) also reported similar growth pattern of mungbean and urdbean genotypes under the delayed or advanced planting.

In all green plants the most fundamental and complicated physiological process is photosynthesis and all of its components are sensitive to stress conditions such as photosynthetic pigments, electron transport chain, carbon dioxide reduction pathways and photosystems; any type of 
Mannan et al. / Intl J Agric Biol, Vol 25, No 3, 2021

Table 4: Effect of planting dates on yield attributes of mungbean cultivars

\begin{tabular}{|c|c|c|c|c|}
\hline Treatments & Number of pods per plant $^{-1}$ & Pod length $(\mathrm{cm})$ & Number of grains per pod & 1000-grain weight (g) \\
\hline \multicolumn{5}{|l|}{ Planting dates (PD) } \\
\hline July 01 & 19.83 & $8.79 \mathrm{~A}$ & $10.46 \mathrm{~A}$ & $53.21 \mathrm{~A}$ \\
\hline August 01 & 18.83 & $8.29 \mathrm{~B}$ & $7.46 \mathrm{~B}$ & $49.46 \mathrm{~B}$ \\
\hline HSD value at $p \leq 0.01$ & NS & 1.33 & 1.33 & 0.01 \\
\hline \multicolumn{5}{|l|}{ Mungbean cultivars (C) } \\
\hline MGP-17 & $22.83 \mathrm{~A}-\mathrm{C}$ & $9.18 \mathrm{D}-\mathrm{F}$ & 7.83 & $51.73 \mathrm{H}$ \\
\hline DM-D4 & $12.16 \mathrm{EF}$ & $6.08 \mathrm{NO}$ & 8.33 & $47.48 \mathrm{~S}$ \\
\hline C5/95-3-31 & $15.83 \mathrm{C}-\mathrm{F}$ & $6.91 \mathrm{~K}-\mathrm{M}$ & 7.83 & $48.12 \mathrm{P}$ \\
\hline C6/95-3-8 & 14.50 D-F & $6.61 \mathrm{~L}-\mathrm{N}$ & 7.50 & $47.94 \mathrm{Q}$ \\
\hline $5-63-94$ & $12.83 \mathrm{EF}$ & $6.28 \mathrm{M}-\mathrm{O}$ & 9.16 & $47.81 \mathrm{R}$ \\
\hline TM-1407 & $10.16 \mathrm{~F}$ & $5.81 \mathrm{O}$ & 7.83 & $47.30 \mathrm{~T}$ \\
\hline MGP-01 & $21.83 \mathrm{~A}-\mathrm{D}$ & $8.81 \mathrm{E}-\mathrm{G}$ & 9.16 & $51.56 \mathrm{I}$ \\
\hline NM-11 & $24.50 \mathrm{AB}$ & $9.38 \mathrm{DE}$ & 6.50 & $51.94 \mathrm{G}$ \\
\hline MGP-41 & $19.83 \mathrm{~A}-\mathrm{E}$ & $8.58 \mathrm{~F}-\mathrm{H}$ & 8.50 & $51.37 \mathrm{~J}$ \\
\hline $5-63-1$ & $15.83 \mathrm{C}-\mathrm{F}$ & $7.18 \mathrm{~J}-\mathrm{L}$ & 8.16 & $48.34 \mathrm{O}$ \\
\hline MGP-16 & 18.16 A-E & $8.38 \mathrm{GH}$ & 8.33 & $50.88 \mathrm{~K}$ \\
\hline NM20-21 & $23.83 \mathrm{AB}$ & $9.81 \mathrm{CD}$ & 10.50 & $53.83 \mathrm{~F}$ \\
\hline MUNG-88 & $16.83 \mathrm{~B}-\mathrm{F}$ & $7.48 \mathrm{I}-\mathrm{K}$ & 8.50 & $48.61 \mathrm{~N}$ \\
\hline NM-121-25 & $25.83 \mathrm{~A}$ & $11.58 \mathrm{~A}$ & 11.16 & $56.47 \mathrm{~A}$ \\
\hline RAMZAN & $16.83 \mathrm{~B}-\mathrm{F}$ & $8.15 \mathrm{G}-\mathrm{I}$ & 9.83 & $50.78 \mathrm{~L}$ \\
\hline NM-2016 & $25.50 \mathrm{~A}$ & $11.28 \mathrm{~A}$ & 11.16 & $56.12 \mathrm{~B}$ \\
\hline NM-19-19 & $24.16 \mathrm{AB}$ & $10.81 \mathrm{AB}$ & 10.16 & $55.81 \mathrm{C}$ \\
\hline 1099 & 19.16 A-E & $7.81 \mathrm{H}-\mathrm{J}$ & 7.83 & $50.43 \mathrm{M}$ \\
\hline NM-51 & $22.16 \mathrm{~A}-\mathrm{D}$ & $10.48 \mathrm{BC}$ & 10.83 & $55.54 \mathrm{D}$ \\
\hline NM13-1 & $23.83 \mathrm{AB}$ & $10.18 \mathrm{BC}$ & 9.16 & $54.70 \mathrm{E}$ \\
\hline HSD value at $p \leq 0.01$ & 7.71 & 0.77 & NS & 0.07 \\
\hline Significance Level (PD) & $* *$ & $* *$ & $* *$ & $* *$ \\
\hline Significance Level (C) & NS & $* *$ & NS & $* *$ \\
\hline Significance Level $(\mathrm{PD} \times \mathrm{C})$ & NS & NS & NS & ** \\
\hline
\end{tabular}

stress at any stage of life affects overall photosynthetic efficiency of green plants (Ashraf and Harris 2013; Sharma et al. 2019). Current study revealed that late sown cultivars had an adverse effect on the physiological attributes of mungbean as compared to optimum planting due to variation in the ambient temperature (Fig 1; Table 3). Higher chlorophyll contents values indicate greater photosynthetic ability of plants. It was seen that chlorophyll contents of mungbean genotypes revealed a significant variation with leaf surface temperature, these results showed are in accordance with previous reports (Guilioni et al. 2003). These results are in conformity with those of Kaleem et al. (2009) who found that different temperatures affect photosynthetic rate differently, that is, photosynthetic rate increased with increase in temperature. Similarly, Baydar and Erbas (2005) concluded that low temperature is one of the limiting factors that adversely affect photosynthesis which is sensitive to cold stress. Similarly, Grulke et al. (2004) who found that the magnitude of stomatal conductance varies temporally with leaves age, from prereproductive to reproductive stage leaf age caused a decline in stomatal conductance in sunflower. These results are also in accordance with those of Orta et al. (2002) who concluded that, as percent soil water decreased, crop water stress index increased causing decrease in stomatal conductance. Baydar and Erbas (2005) concluded that low temperature is one of the limiting factors that adversely affect crop hydraulic and physiological processes i.e. stomatal conductance, sub-stomatal $\mathrm{CO}_{2}$ concentration, photosynthetic rate, transpiration rate and water use efficiency. These results are in conformity with those of Bunce (2007) who concluded that hydraulic conductance in plants is affected by environmental factors. In the past studies, it has been reported that in rice seedlings there was greater biomass production due to high water use efficiency and reduced transpiration rate, ultimately higher photosynthetic rate (Karaba et al. 2007). In present study the genotypes with higher transpiration rate showed less water use efficiency (Table 3). However, some genotypes exhibit less transpiration and greater water use efficiency resultantly higher photosynthetic rate and biomass production and withstand under delayed planting conditions.

Leaf temperature is an important parameter in physiological life of crop plants. It directly affects photosynthesis and water use efficiency; ultimately controls all growth stages (Brooks and Farquhar 1985; Lohani et al. 2020). In this experiment genotypes varied significantly in leaf temperature. Beyond the optimum limit leaf temperature (optimum planting time) inhibits the photosynthetic rate by stimulating photorespiration and cause damages to photosynthetic apparatus (Schrader et al. 2004; Rasmusson et al. 2020). Rubisco activity is reduced at moderate elevation in leaf temperature resultantly reduce photosynthetic rate (Salvucci et al. 2001; Wi et al. 2020). All these studies showed that photosynthesis and water use efficiency are leaf temperature dependent attributes.

In the present studies, result showed that late sown had an adverse effect on the yield attributes of mungbean as 
compared to optimum planting time (Table 4). Soomro (2003) reported that delay in sowing causes a substantial decrease in all the growth and development parameters of mungbean. The highest seed yield obtained from optimum planting might be due to suitable temperature prevailing accompanied by higher soil moisture content due to sufficient rainfall, which enhanced the vegetative as well as reproductive growth of the crop. Relatively higher grain yield from optimum sowing was probably due to higher grain yield plant ${ }^{-1}$ and its attributes with number of pods plant $^{-1}$, grains pod $^{-1}$ and test weight (Singh et al. 2010; Khanum et al. 2019). Differential response of different varieties was also observed by Singh et al. (2010) and Sadeghipour (2008). For obtaining higher mungbean grain yield, not only vegetative growth and development but efficient utilization of photosynthates towards economic sink enlargement is also important (Reddy 2009; Singh et al. 2010; Khanum et al. 2019). Response to normal planting date also revealed significantly higher accumulation of total dry matter $\left(\mathrm{g} \mathrm{plant}^{-1}\right)$ in normal sowing than late sowing and this might have resulted in production of higher biological yield in normal planting date (Ramakrishna et al. 2000; Reddy 2009; Khanum et al. 2019).

\section{Conclusion}

In crux, delayed planting significantly reduced the germination, growth, physiological and yield attributes of mungbean cultivars; though the cultivars differ in their response. Overall NM-121-25 and NM-2016 performed better as compared to rest of mungbean cultivars, and DMD4 and TM-1407 cultivars performed poorly. Mungbean cultivars NM-121-25 and NM-2016 can be sown in late sown conditions to get higher yield.

\section{Authors Contributions}

$\mathrm{AM}$ and MBC Planned the whole work. AM performed the experiments and MS helped to analyses the DATA.

\section{References}

Abdelmageed AHA, N Gruda (2009). Performance of different tomato genotypes in the arid tropics of Sudan during the summer season. II. Generative development. J Agric Rural Develop Trop Subtrop 110:147-154

Aditya P, K Jitendra (2011). Biology and Breeding of Food Legumes. Michigan State University, East Lansing, Michigan, USA

Ahmad MSA, M Hossain, S Ijaz, AK Alvi (2008). Photosynthetic performance of two mungbean (Vigna radiata) cultivars under lead and copper stress. Intl J Agric Biol 10:167-172

Ali M, S Gupta (2012). Carrying capacity of Indian agriculture: Pulse crops. Curr Sci 102:874-881

Ali S, AH Shah, R Gul, H Ahmad, H Nangyal, SK Sherwin (2014). Morpho-Agronomic Characterization of Okra (Abelmuscus esculentus L.). World App Sci J 31:336-400

Ashraf M, PJ Harris (2013). Photosynthesis under stressful environments: An overview. Photosynthetica 51:90-163

Asseng S, IAN Foster, NC Turner (2011). The impact of temperature variability on wheat yields. Global Change Biol 17:997-1012
Association of Official Seed Analysts (AOSA) (1990). Rules for testing seeds. J Seed Technol 12:1-112

Baydar H, S Erbas (2005). Influence of seed development and seed position on oil, fatty acids and total tocopherol contents in sunflower (Helianthus annus L.). Turk J Agric 29:179-186

Brooks A, GD Farquhar (1985). Effects of temperature on the $\mathrm{O}_{2} / \mathrm{CO}_{2}$ specificity of ribulose-1,5-bisphosphate carboxylase/oxygenase and the rate of respiration in the light. Estimates from gas exchange measurements on spinach. Planta 165:397-406

Bunce JA (2007). Low carbon dioxide concentrations can reverse stomatal closure during water stress. Physiol Plantarum 130:552-559

Campbell, J Brian, FA Berrada, C Hudalla, S Amaducci, JK McKay (2019). Genotype $\times$ environment interactions of industrial hemp cultivars highlight diverse responses to environmental factors. Agrosys Geosci Environ 2:1-11

Chauhan YS, C Douglas, RCN Rachaputi, P Agius, W Martin, A Skerman (2010). Physiology of mungbean and development of the mungbean crop model. In: Proc of the $1^{\text {st }}$ Australian Summer Grains Conference, pp:21-24. Gold Coast, Australia

Ellis RA, EH Roberts (1981). The quantification of ageing and survival in orthodox seeds. Seed Sci Technol 9:373-409

Gezer B (2018). Adsorption capacity for the removal of organic dye pollutants from wastewater using carob powder. Intl J Agric For Life Sci 2:1-14

Grulke NE, R Alonso, T Nguyen, C Cascido, W Dobrowolski (2004). Stomata open at night in pole-sized and mature ponderosa pine. Tree Physiol 24:1001-1010

Guilioni L, J Wery, J Lecoeur (2003). High temperature and water deficit may reduce seed number in field pea purely by decreasing plant growth rate. Funct Plant Biol 30:1151-1164

Haider MU, M Hussain, M Farooq, A Nawaz (2020). Optimizing zinc seed priming for improving the growth, yield and grain biofortification of mungbean (Vigna radiata (L.) wilczek). J Plant Nutr 43:1438-1446

Howarth CJ (2005). Genetic improvements of tolerance to high temperature. In: Abiotic Stresses Plant Resistance through Breeding and Molecular Approaches, Ashraf M, PJC Harris (eds.). Howarth Press, New York, USA

Hussain MM, LK Balbaa, MS Gaballah (2007). Salicylic acid and salinity effects on growth of maize plants. Res J Agric Biol Sci 3:321-328

Hussain M, M Farooq, G Shabir, MB Khan, AB Zia (2012a). Delay in planting decreases wheat productivity. Intl J Agric Biol 14:533-539

Hussain M, G Shabir, M Farooq, K Jabran, S Farooq (2012b). Developmental and phenological responses of wheat to sowing dates. Pak J Agric Sci 49:459-468

Kaleem S, F Hassan, A Saleem (2009). Influence of environmental variations on physiological attributes of sunflower. Afr J Biotechnol 8:23-32

Karaba A, S Dixit, R Greco, A Aharoni, KR Trijatmiko, N MarschMartinez, A Pereira (2007). Improvement of water use efficiency in rice by expression of HARDY, an Arabidopsis drought and salt tolerance gene. Proc Natl Acad Sci USA 104:15270-15275

Khanum MM, MM Bazzaz, MA Hossain, MS Huda, M Nuruzzaman (2019). Effect of sowing date on performances of mungbean at Bari research field in Dinajpur. Bangl J Environ Sci 37:52-55

Lohani N, MB Singh, PL Bhalla (2020). High temperature susceptibility of sexual reproduction in crop plants. J Exp Bot 71:555-568

Miah MAK, MP Anwar, M Begum, AS Juraimi MA Islam (2009). Influence of sowing date on growth and yield of summer mungbean varieties. J Agric Soc Sci 5:73-76

Miklas PN, SP Singh (2007). Pulses, Sugar and Tuber Crops. Pulses, Sugar and Tuber Crops. Michigan State University, East Lansing, Michigan, USA

Naika S, J Juede, M Goffau, M Hilmi, V Dam (2005). Cultivation of Tomato: Production, Processing and Marketing (Rev. edn.). Agrodok series No. 17. Agromisa/CTA, Wageningen, The Netherland

Nassar NMA (2003). Cassava, Manihot esculent Crantz genetic resources: VI. Anatomy of a diversity center. Genet Mol Res 2:214-222

Naveed M, M Shafiq, CM Rafiq, MS Saeed (2015). Planting date effects on the incidence of mungbean yellow mosaic virus (MYMV) and cultivars performance under rainfed environments. Plant Knowl $J$ Southern Cross Pub Group 4:7-12 
Orta AH, T Erdem, Y Erdem (2002). Determination of water stress index in sunflower. Helia 37:27-38

Palsaniya S, R Puniya, A Sharma, BR Bazaya D Kachroo (2016). Effect of sowing dates and varieties on growth, yield and nutrient uptake of summer mungbean (Vigna radiata). Ind J Agron 61:256-258

Phurailatpam AK, AK Pal, S Singh (2007). Growth pattern and its impact on seed yield in cultivated and wild genotypes of Vigna. J Food Legum 20:161-64

Ramakrishna A, CLL Gowda, C Johansen (2000). Management factors affecting legumes production in the Indo-Gangetic Plain. In: Legumes in Rice and Wheat Cropping Systems of the Indo-Gangetic Plain-Constraints and Opportunities, pp:156-165. Johansen C, JM Duxbury, SM Virmani, CLL Gowda (eds.). ICRISAT, Patancheru, Andhra Pradesh, India

Rasmusson LM, P Buapet, R George, M Gullström, PC Gunnarsson, M Björk (2020). Effects of temperature and hypoxia on respiration, photorespiration, and photosynthesis of seagrass leaves from contrasting temperature regimes. ICES J Marine Sci 77:2056-2065

Reddy AA (2009). Pulses production technology: Status and way forward. Eco Polit Weekly 44:73-80

Sadeghipour O (2008). Response of mungbean varieties to different sowing dates. Pak J Biol Sci 11:2048-2050

Salvucci ME, KW Osteryoung, SJ Crafts-Brandner, E Vierling (2001). Exceptional sensitivity of Rubisco activase to thermal denaturation in vitro and in vivo. Plant Physiol 127:1053-64

Sarwar MA, SR Malik, W Ahmad, MS Mahmood, M Jawad, M Asadullah, I Ahmad, M Imran (2019). Production efficiency of promising mungbean genotypes affected by different sowing dates under rainfed conditions. Pak J Agric Res 32:52-58
Schrader SM, RR Wise, WF Wacholtz, DR Ort, TD Sharkey (2004). Thylakoid membrane responses to moderately high leaf temperature in Pima cotton. Plant Cell Environ $27: 725-735$

Sharma A, V Kumar, B Shahzad, M Ramakrishnan, GPS Sidhu, AS Bali, N Handa, D Kapoor, P Yadav, K Khanna, P Bakshi (2019). Photosynthetic response of plants under different abiotic stresses: A review. J Plant Growth Regul 1-23

Singh AK, N Chandra, RC Bharati, SK Dimree (2010). Effect of seed size and seeding depth on Faba bean (Vicia faba L.) productivity. Environ Ecol 28:1722-1727

Somroo AH, MA Aaraen, M Khaelski, B Bhutto (2003). Comparative study on the Physico-chemical Composition of Industrial yogurt and ingenious dahi. $J$ Biol Sci 3:86-90

Steel RGD, JH Torrie, DA Dickey (1997). Principles and Procedures of Statistics: A Biometrical Approach, $3^{\text {rd }}$ edn. McGraw Hill Book Inc. Co., New York, USA

Thakur P, S Kumar, JA Malik, JD Berger, H Nayyar (2010). Cold stress effects on reproductive development in grain crops: An overview. Environ Exp Bot 3:429-443

Uddin MS, MM Rahman, MM Hossain, MAK Mian (2014). Genetic diversity in eggplant genotypes for heat tolerance. SAARC J Agric 12:25-39

Vorasoot N, P Songsri, C Akkasaeng, S Jogloy, A Patanothai (2003). Effect of water stress on yield and agronomic characters of peanut. $J \mathrm{Sci}$ Technol 3:283-288

Wi SH, HJ Lee, S An, SK Kim (2020). Evaluating growth and photosynthesis of kimchi cabbage according to extreme weather conditions. Agronomy 10; Article 1846 